\title{
Secondary impacts of COVID-19: Risk of vaccination reduction and global resurgence of measles
}

Tasmiah Nuzhath ${ }^{1}$, Md Mahbub Hossain ${ }^{2}$

${ }^{1}$ Department of Population Sciences, University of Dhaka, Dhaka, Bangladesh

${ }^{2}$ Nature Study Society of Bangladesh, Khulna, Bangladesh

Correspondence: nuzhathtasmiah33@gmai.com

Coronavirus disease (COVID-19) was declared a pandemic by the World Health Organization on March 11, 2020. ${ }^{1}$ The pandemic will adversely impact routine immunization globally as routine vaccination services provision has been significantly affected at least 68 countries and an estimated 80 million children under the age of 1 living in these countries, thereby increasing the likelihood of vaccine-preventable diseases including measles outbreak in the absence of prompt actions. ${ }^{2}$

The World Health Organization's Strategic Advisory Group of Experts (SAGE) on Immunization has temporarily suspended all mass vaccination campaigns and the national lockdown across the countries has resulted in the postponement of routine immunization programs following the recommendations of maintaining physical distance. ${ }^{34}$ This is a major concern as 37 low and middle income countries, some of which are battling ongoing measles outbreaks, have suspended routine immunization activities and 117 million children are at risk of not receiving measles vaccination. ${ }^{5}$ These countries are Mexico, Honduras, Colombia, Bolivia, Chile, Paraguay, Brazil, Dominican Republic, Chad, Nigeria, Guinea, Central African Republic, Equatorial Guinea, Gabon, Democratic Republic of Congo, Angola, Namibia, Botswana, Eswatini, Zambia, Comoros, Kenya, Somalia, Ethiopia, Djibouti, South Sudan, Lebanon, Ukraine, Uzbekistan, Kazakstan, Krygztan, Nepal, Bangladesh, Maldives, Cambodia, Philippines and Vietnam. ${ }^{6}$ Some of these countries such as DRC Congo, Brazil, Phillipines, Kazakstan, Bangladesh, Nigeria, and Ethiopia have reported the highest number of measles cases globally as of March, 2020, while some of the them are wartorn African nations in the Sahel including Central African Republic, Chad, South Sudan, and Somalia that have reported significant increase in the number of measles deaths in 2019. ${ }^{5,7}$

Gaps in immunization in high-income countries like the US, UK and France led to alarming measles outbreaks in 2019 and the impact of COVID-19 will exacerbate the situation. Demand for immunization has decreased during COVID-19 in these regions where parents visit the clinic or private pediatric offices for routine inoculation. This has led to reduction in the immunization rates as parents avoid doctor's visits to maintain physical distancing and to prevent children from being infected by COVID-19. ${ }^{8}$ Several states in the including Massachusetts, Michigan and Minnesota have reported a decrease in the uptake of measles vaccination. ${ }^{8}{ }^{8}$ According to data from PCC, an electronic health records company for pediatricians, information from 1000 independent pediatrician showed that measles vaccination uptake dropped by $50 \%$ from the week of 16 February to the week of 5 April 2020 in the United States. ${ }^{8}$ Data for routine pediatric vaccines from Vaccines for Children Program (VFC) and Vaccine Safety Datalink (VSD) from January 6April 19, 2020 found sharpest decline in measles immunization uptake in the US. ${ }^{10}$ Any disruption of immunization services, even for short duration will result in an increased likelihood of vaccine preventable disease such as measles outbreaks. ${ }^{11}$ 
Furthermore, there will be disruption in the supply and distribution of vaccinations globally as a result of the border closures and disruption to flights and transportation to mitigate the transmission of the virus. ${ }^{12} 21$ developing countries have reported shortages in vaccination as funds are being allocated to support health systems acquire essential diagnostics and necessary equipment to combat coronavirus and thus less money is available for countries to acquire vaccinations. ${ }^{3,10}$ Delivery of routine services such as immunization will also be affected due to shortage of resources as healthcare providers are deployed to tackle COVID-19.

These data suggest a suboptimal coverage of vaccination programs, which may critically affect the existing vaccination goals in many countries while threatening the population health with inadequate control of measles. Amidst ongoing COVID-19 pandemic, it is therefore, essential to prevent these challenges through effective policymaking and strategic planning. Such measures should not only aim to recover the gaps in national and regional immunization goals but also emphasize on building health systems resiliency to external shocks that may affect vaccination programs across context.

Measures should be taken to conduct situational analyses in different high-risk countries to evaluate the gaps in terms of vaccination coverage and identify vulnerable groups to develop tailored vaccination strategies and operational plans to address immunization gaps and reach every child with vaccines. Efforts to intensify population-based immunization record and vaccine preventable disease surveillance at sub-national level as part of integrated health information system will help to support routine immunization decision-making. Countries will need to increase community-level partnership to mobilize resources to ensure effective vaccination coverage. Community engagement will help to enhance awareness in the community and increase demand for vaccination by addressing misconceptions and increasing the understanding of the importance of routine immunization. Consequently, integrating field-level COVID-19 responses with vaccination efforts will ensure cost-effective and optimal prevention of COVID-19 as well as restoration of vaccination gaps in respective countries and reduce infectious disease burden on the health system. In order to maximize reach, pulse immunization strategy can be implemented to improve uptake among the refugees and other marginalized population. Responses should include utilizing extensive media coverage and encouraging physicians to promote catch up immunization and enhance demand for immunization among primary care givers.

Lastly, monitoring, accountability, and leadership are essential to address the ongoing crisis as well as improve health system responses to vaccination programs through integrated, coordinated, and collaborative initiatives. The current COVID-19 pandemic appears to be a threat to global achievements in measles control, which necessitates stronger measures to develop resilience in vaccination programs in the future.

\section{Declaration of interests}

We declare no competing interest. 


\section{References}

1. World Health Organization. Director-General's opening remarks at the media briefing on COVID-19. 2020 Mar 11. [cited 2020 Apr 11].

Available from: https://www.who.int/dg/speeches/detail/who-director-general-s-openingremarks-at-the-media-briefing-on-covid-19---11-march-2020

2. World Health Organization. At least 80 million children under one at risk of diseases such as diphtheria, measles and polio as COVID-19 disrupts routine vaccination efforts, warn Gavi, WHO and UNICEF. 2020 May 22. [cited 2020 May 31] https://www.who.int/news-room/detail/22-05-2020-at-least-80-million-children-underone-at-risk-of-diseases-such-as-diphtheria-measles-and-polio-as-covid-19-disruptsroutine-vaccination-efforts-warn-gavi-who-and-unicef

3. World Health Organization. Guiding principles for immunization activities during the COVID-19 pandemic. Interim guidance 26 March 2020. 2020 Mar 26. [cited $2020 \mathrm{Apr}$ 11]. Available from: https://apps.who.int/iris/bitstream/handle/10665/331590/WHO2019-nCoV-immunization_services-2020.1-eng.pdf

4. GAVI The Vaccine Alliance. COVID-19: massive impact on lower-income countries threatens more disease outbreaks. 2020 Apr 3. [cited 2020 Apr 11]. Available from: https://www.gavi.org/news/media-room/covid-19-massive-impact-lowerincome-countries-threatens-more-disease-outbreaks

5. Centers for Disease Control and Prevention. Global Measles Outbreaks. 2020 Mar 6. [cited 2020 Apr 11]. Available from: https://www.cdc.gov/globalhealth/measles/globalmeaslesoutbreaks.htm

6. Measles \& Rubella Initiative. More than 117 million children at risk of missing out on measles vaccines, as COVID-19 surges. 2020 April 14. [cited 2020 May 7].

Available from:https://measlesrubellainitiative.org/measles-news/more-than-117-millionchildren-at-risk-of-missing-out-on-measles-vaccines-as-covid-19-surges/

7. Hotez, P. J., Nuzhath, T., \& Colwell, B. Combating vaccine hesitancy and other 21st century social determinants in the global fight against measles. Current Opinion in Virology 2020, 41, 1-7.

8. Hoffman J. Vaccine Rates Drop Dangerously as Parents Avoid Doctor's Visits. NY Times. 2020 Apr 23. [cited 2020 May 7]. Available from: https://www.wowt.com/content/news/Children-missing-vaccinations-as-parents-skip-8. doctor-visits-during-pandemic-569905681.html

9. Bramer CA, Kimmins LM, Swanson R, et al. Decline in Child Vaccination Coverage During the COVID-19 Pandemic - Michigan Care Improvement Registry, May 2016May 2020. MMWR Morb Mortal Wkly Rep. ePub: 18 May 2020. DOI: http://dx.doi.org/10.15585/mmwr.mm6920elexternal icon

10. Hotez PJ. COVID19 meets the Antivaccine Movement. Microbes and Infection. 2020 May 19. 
11. Suk JE, Jimenez AP, Kourouma M, Derrough T, Baldé M, Honomou P, et al. Post-Ebola measles outbreak in Lola, Guinea, January-June 2015. Emerging infectious diseases 2016;22(6):1106.

12. GAVI The Vaccine Alliance. Gavi Board calls for bold engagement to respond to COVID-19. 2020 Mar 21. [cited 2020 Apr 11]. Available from:

https://www.gavi.org/news/media-room/gavi-board-calls-bold-engagement-respondcovid-19 